\title{
Implementing Edge Detection for Detecting Neurons from Brain to Identify Emotions
}

\author{
Madhulika \\ Assistant Professor, \\ Department of Computer Science Engineering \\ Amity University \\ Noida, India \\ Amandeep \\ Assistant Professor, \\ Department of Computer Science Engineering \\ Amity University \\ Noida, India
}

\author{
Abhay Bansal, PhD. \\ Professor, Head \\ Department of Computer Science Engineering \\ Amity University \\ Noida,India
}

\author{
Madhurima \\ Assistant Professor, \\ Department of Computer Science Engineering \\ Amity University \\ Noida, India
}

\begin{abstract}
Edges of an image are considered a type of crucial information that can be extracted by applying detectors with different methodology. Edge detection is a basic and important subject in computer vision and image processing In this Paper we discuss several Digital Image Processing Techniques applied in edge feature extraction. Firstly, Linear filtering of Image is done is used to remove noises from the image collected. Secondly, some edge detection operators such as Sobel, Log edge detection, canny edge detection are analyzed and then according to the simulation results, the advantages and disadvantages of these edge detection operators are compared. It is shown that the canny operator can obtain better edge feature. Finally, Edge detection is applied to identify neurons in Brain. After this the Neurons are classified and feature vector will be calculated.
\end{abstract}

Keywords- Filters, Sobel, Canny, Log, Distortion, Edge Detection Introduction (Heading 1)

\section{INTRODUCTION}

Detecting edges is types of Image Segmentation Techniques which shows the presence of edges or line in an image and give them boundaries in an appropriate way [1].The edge is a set of those pixels that exists between object and background, object and object, region and region, and between element and element. Edge always exists with different grey level in two neighboring areas .Edge detection based on range noncontinuity. Image edge detection is one of the important contents in the image processing and analysis, and also is a kind of issues which are unable to be resolved completely so far [2]. When image is acquired, there are so many factors like projection, mix, aberrance and noise are produced. These factors effect on image feature's making it blur and distortion, consequently it is very difficult to extract image feature. Moreover, due to such factors it is also difficult to detect edge.

The method of image edge detection and extraction is very hot research topic in the domain of image processing and analysis technique. Edge feature extraction has been applied in many areas widely. This paper mainly discusses about several edge detection operators and applied in the detecting Neurons in it .Firstly the acquired image is filtered and denoised. In the process of denoising, Linear Filter is used. And then different operators are applied to detect edge including Sobel operator, Log operator, Canny operator. Finally the edge detection is applied to detect neurons in human brain.

\section{LOAD IMAGE IN MATLAB}

A Loading Original image in Matlab

The image is loaded in Matlab by GUI developed using GUIDE tool. The original image of a Neuron is loaded using a load module.

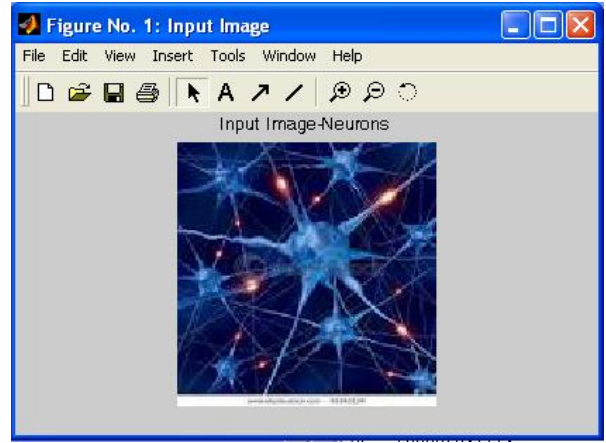

Figure 1 Original image of a Neuron in Human Brain

\section{IMAGE FILTERING}

\section{- $\quad$ Linear filter}

The Image is filtered using Low pass and high pass filter. Low pass filtering, It is also called "smoothing". It helps us to employ or to remove high spatial frequency noise from a digital image. Noise is often introduced during the analog-todigital conversion process as a side-effect of the physical conversion of patterns of light energy into electrical patterns [3]. Smoothing is done in Low Pass Filtering. In Edges are detected using High Pass Filtering. The original image is loaded then filtered using imfilter function in Matlab. 


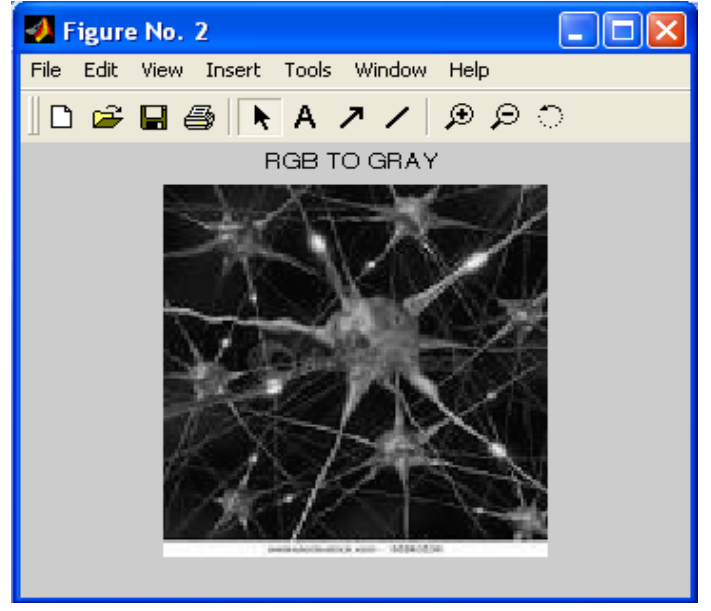

Figure 2 Original Image RGB to GRAY Conversion

\section{Edge Detection}

Edge detection is very important in the digital image processing, because the edge is boundary of the target and the background. With the help of edges we can differentiate the target and the background. The basic idea of image detection is to outstand Using edge detection we can outstand partial edge of the image with the help of edge enhancement operator firstly. After that we define the 'edge intensity' of pixels and extract the set of edge points through setting threshold.The borderline detected may produce interruption as a result of existing noise and image dark. Thus edge detection contains the following two parts:

- The edge points set are extracted using edge operators.

- Some edge points in the edge points set are removed and a number of edge points are filled in the edge points set.

Then the obtained edge points are connected to be a line. The common used operators are the Sobel, Log, Canny operators [4].

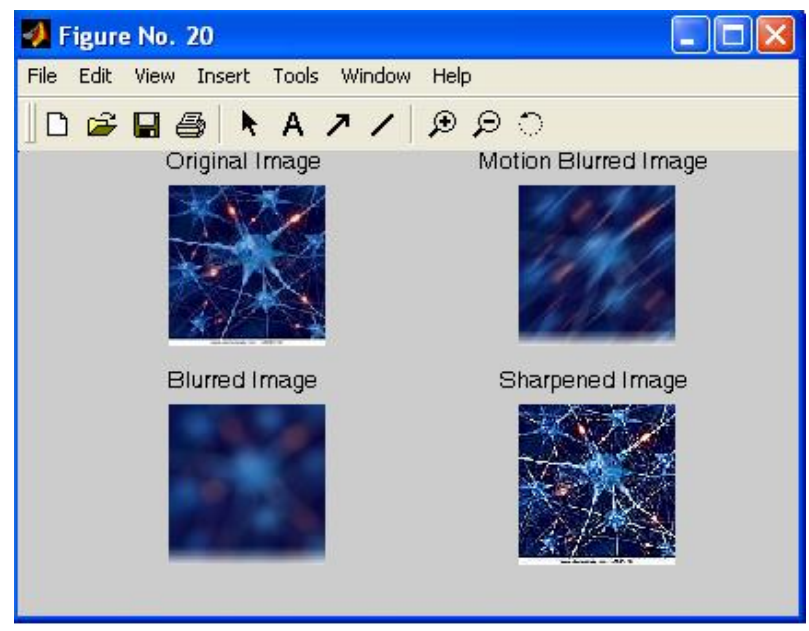

Figure 3 Sharpened Images

\section{- Sobel Operator}

Standard Sobel operators, for a $3 \times 3$ neighborhood, each simple central gradient estimate is vector sum of a pair of orthogonal vectors [1]. Each orthogonal vector is a directional derivative estimate multiplied by a unit vector specifying the derivative's direction. The vector sum of these simple gradient estimates amounts to a vector sum of the 8 directional derivative vectors. Thus for a point on Cartesian grid and its eight neighbors having density values as shown:

\begin{tabular}{|l|l|l|}
\hline$a$ & $b$ & $C$ \\
\hline$d$ & $e$ & $f$ \\
\hline$g$ & $h$ & $i$ \\
\hline
\end{tabular}

In [6], the directional derivative estimate vector $G$ was defined such as density difference / distance to neighbor. The direction of $G$ will be given by the unit vector to the approximate neighbor. Note that, the neighbors group into antipodal pairs: (a,i), (b,h), (c,g), (f,d). The vector sum for this gradient estimate:

$G=\frac{(C-G)}{R} \cdot \frac{[1,1]}{R}+\frac{(a-i)}{R} \cdot \frac{[-1,1]}{R}+(\mathrm{b}-\mathrm{h}) \cdot[0,1]+(\mathrm{f}-\mathrm{d}) \cdot[1,0]$

where, $R=\sqrt{ } 2$. This vector is obtained as

$G=[(c-g-a+i) / 2+\mathrm{f}-\mathrm{d},(\mathrm{c}-\mathrm{g}+\mathrm{a}-\mathrm{i}) / 2+\mathrm{b}-\mathrm{h}]$

Here, this vector is multiplied by 2 because of replacing the divide by 2 . The resultant formula is given as follows (see, for detail [1]):

$G^{\prime}=2 . G=[(c-g-a+i)+2 . f-d),(c-g+a-i)$
$+2 .(b-h)]$

The following weighting functions for $\mathrm{x}$ and $\mathrm{y}$ components were obtained by using the above vector is shown in Figure 4

Table 1 Sobel Operator Template

\begin{tabular}{|c|c|c|}
\hline 1 & 0 & 1 \\
\hline-2 & 0 & 2 \\
\hline-1 & 0 & 1 \\
\hline
\end{tabular}

\begin{tabular}{|c|c|c|}
\hline 1 & 2 & 1 \\
\hline 0 & 0 & 0 \\
\hline-1 & -2 & -1 \\
\hline
\end{tabular}

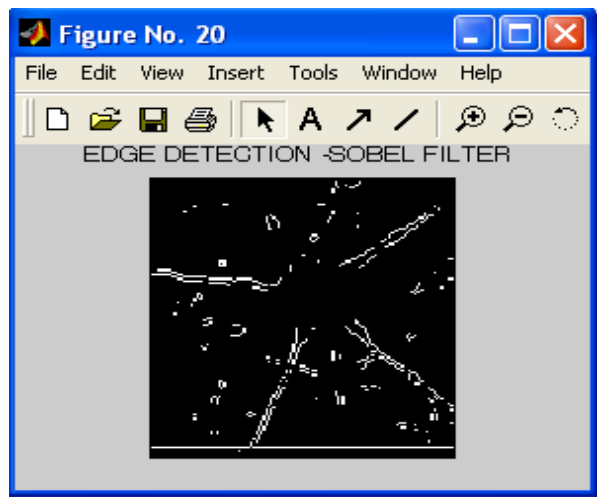

Figure 4 Neurons detected Threshold value 0.041000 


\section{- Log Operator}

In [7], The Log operator is a linear and time-invariant operator. Log operator detects points through searching for spots which two-order differential coefficient is zero in the image grey levels. For a continuous function $f(x, y)$, the $\log$ operator is defined as at point $\mathrm{x}, \mathrm{y}$ :

$$
\Delta^{2} f=\frac{\partial^{2} f}{\partial x^{2}}+\frac{\partial^{2} f}{\partial y^{2}}
$$

The Log operator filters and count differential coefficient for the image. It determines the zero overlapping position of filter output using convolution of revolving symmetrical Log template and the image. The Log operator's template is shown in Figure 7

\begin{tabular}{|l|l|l|}
\hline 0 & -1 & 0 \\
\hline-1 & 4 & -1 \\
\hline 0 & -1 & 0 \\
\hline
\end{tabular}

\begin{tabular}{|l|l|l|}
\hline-1 & -1 & -1 \\
\hline-1 & 8 & -1 \\
\hline-1 & -1 & -1 \\
\hline
\end{tabular}

Table 2 Log Operator template

Log operator , firstly Pre-smooth the image with Gauss lowpass filter, and then find the steep edge in the image making use of the Log operator. Finally we carry on Binarization with zero grey level to give birth to closed, connected outline and eliminate all internal spots. But double pixels boundary usually appears using the Log operator to detect edge, and the operator is very sensitive to noise. So the Log operator is often employed to judge that edge pixels lie in either bright section or dark section of the Image.

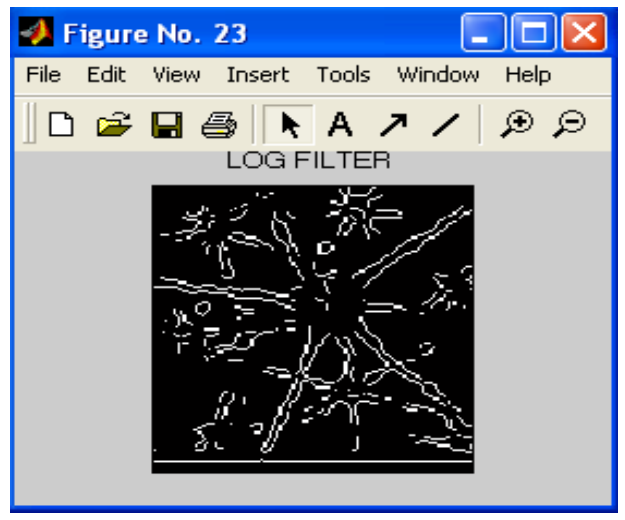

Figure 5 Neurons detected with Log Filter Threshold Value 0.003500

\section{- Canny Filter}

The Canny operator has good performance of detecting edge, which has a wide application. The Canny operator edge detection is to Search for the partial maximum value of image gradient. The gradient is counted by the derivative of Gauss filter. The Canny operator detect strong edge and weak edge using two thresholds. And only when strong edge is connected with weak edge, weak edge will be contained in the output value [8] The theory basis of canny operator is shown in equations.

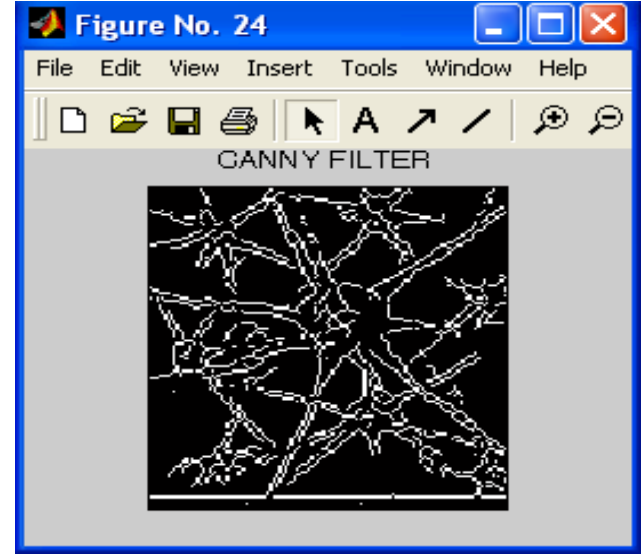

Figure 6 Neurons detected Threshold value 0.0231000

Gauss: $\mathrm{g}(\mathrm{x}, \mathrm{y})=\exp \left[-\left(\mathrm{x}^{2}+\mathrm{y}^{2}\right) / 2 \sigma^{2}\right.$

Edge normals: $\mathrm{n} \perp=\nabla(\boldsymbol{g} * \boldsymbol{p}) /|\boldsymbol{\nabla}(\boldsymbol{g} * \boldsymbol{p})|$

Edge strengths: $\mathrm{G}_{\mathrm{n}} \mathrm{P}=\frac{\partial}{\partial n}[g * p]$

Maximal strengths: $0=\frac{\partial}{\partial n^{\perp}} \mathrm{G}_{\mathrm{n}} \mathrm{P}=\frac{\partial}{\partial n^{\perp}}{ }_{2}[\mathrm{~g} * \mathrm{P}]$

\section{SIMULATIVE RESULTS ANALYSIS}

In order to know about the advantages and disadvantages of these edge detection operators, we detect edge using these different operators respectively. The simulation results are shown in Figure 8

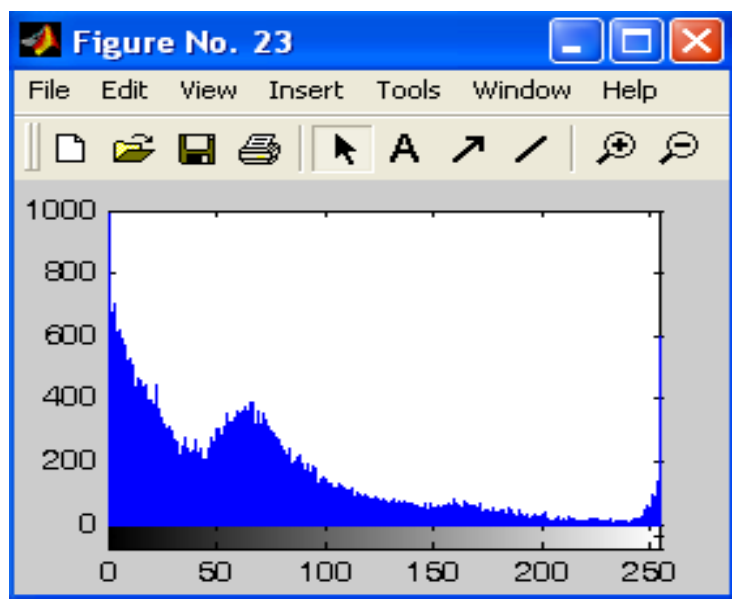

Figure 7 Histogram Generated 

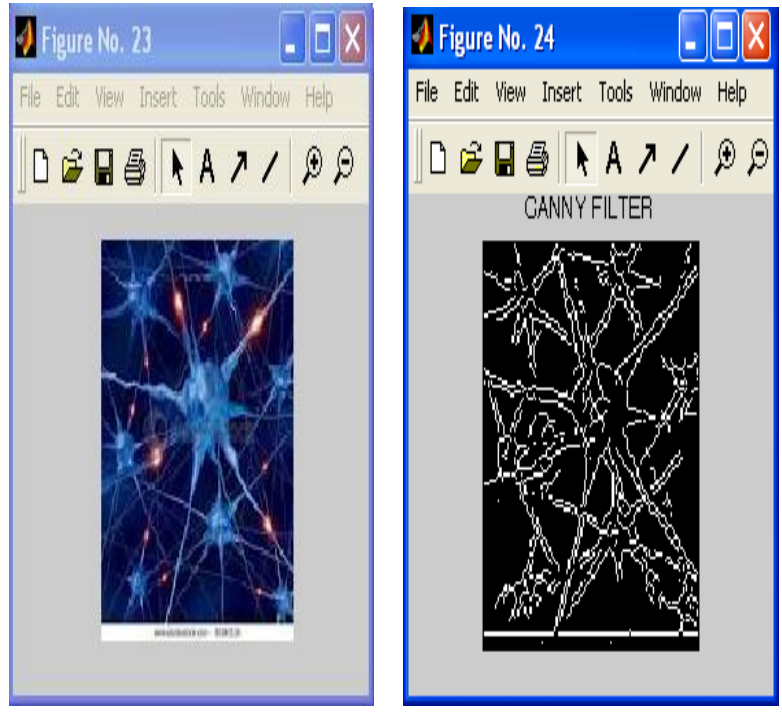

Figure 8 Original Image with Neurons detected

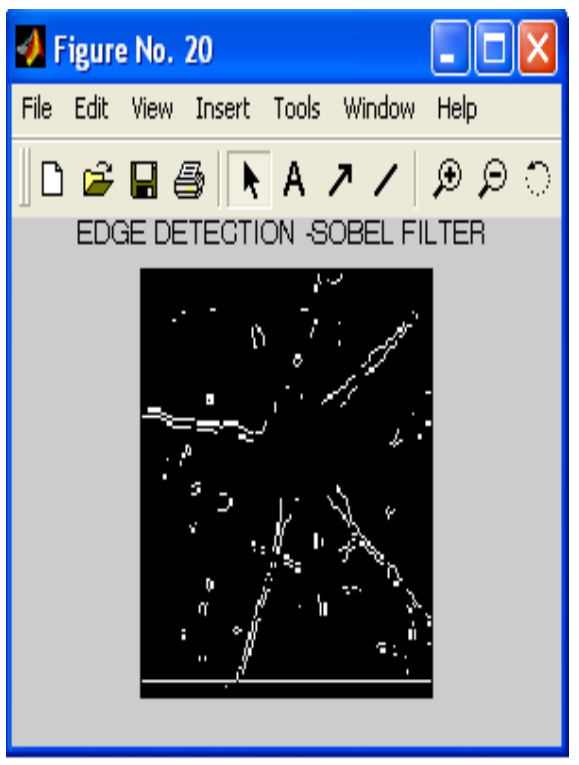

Figure 9 Neurons detected with Sobel Filter Threshold Value 0.036000

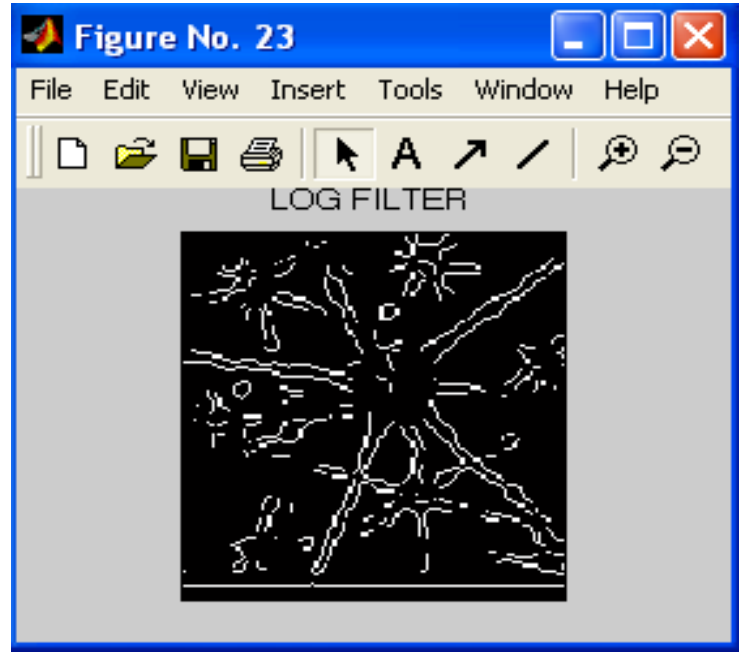

Figure 10 Neurons detected with log Filter Threshold Value $\mathbf{0 . 0 8 5 0 0 0}$

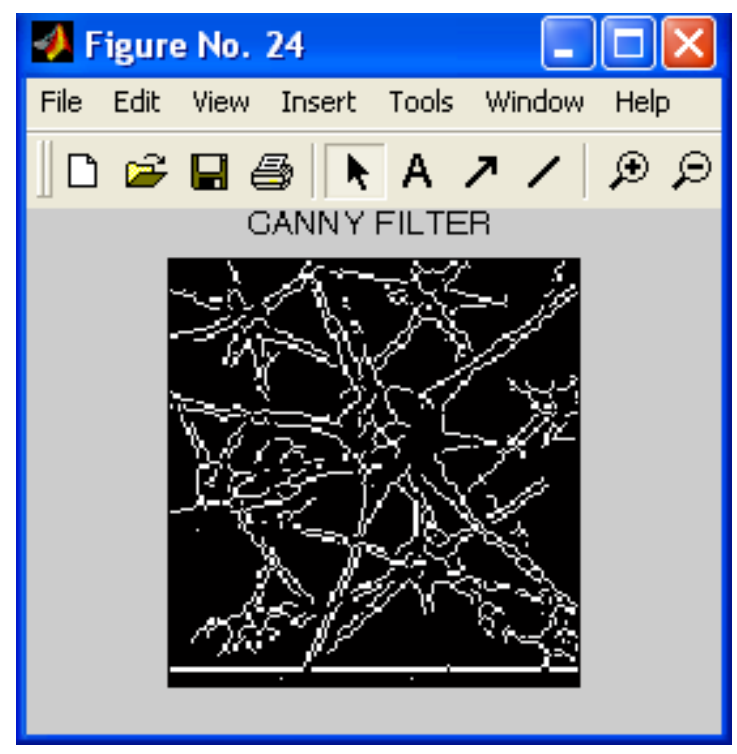

Figure 11 Neurons detected with Canny Filter Threshold Value 0.105000

\section{Segmentation}

In multidimensional signal processing feature extraction and image components classification form a fundamental problem in many applications. Texture is characterized by the spatial distribution of gray levels in a neighborhood. An image region has a constant texture if a set of its local properties in that region is constant, slowly changing or approximately periodic. Texture analysis is one of the most important techniques used in analysis. There are three primary issues in texture analysis: classification, segmentation and shape recovery from texture. Analysis of texture [10] requires the identification of proper attributes or features that differentiate the textures of the image. 


\section{Watershed Transform}

In geography a watershed is the ridge that divides areas drained by different river systems.. The watershed transform applies these ideas to the gray-scale image processing to enable solution of a variety of image segmentation problems. Understanding the watershed transform requires us to consider a gray-scale image as a topological surface, where the values of $f(x, y)$ are interpreted as heights. The watershed transform finds the catchment basins and ridge lines in such a grayscale image. In terms of the problem related to image segmentation the key concept is to change the starting image into another one whose catchment basins are the objects or regions we want to identify as studied by $[11,12]$ for instance.

- Load Image

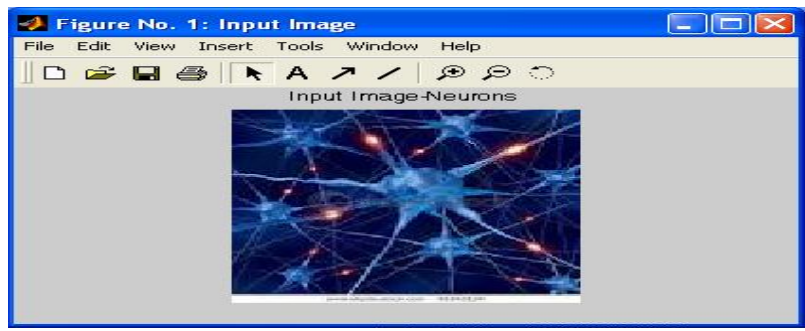

Figure 12 Load Image

- Contrast Enhancement

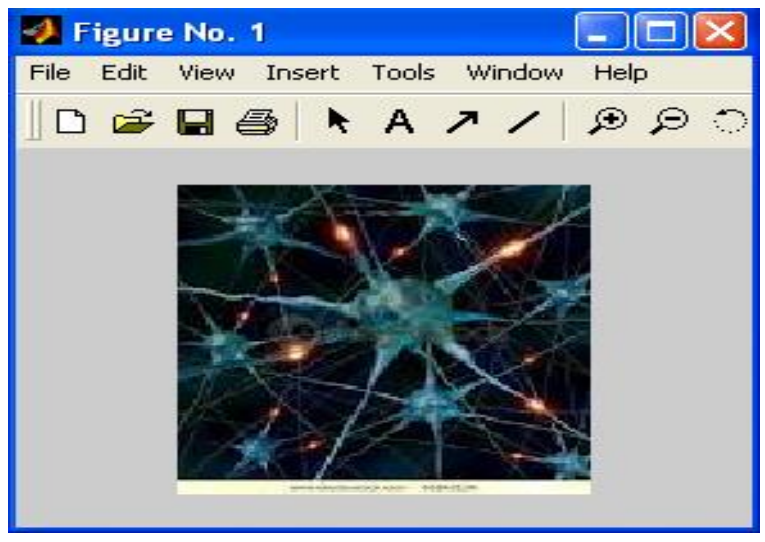

Figure 13 Contrast Enhancement

- Subtract Images

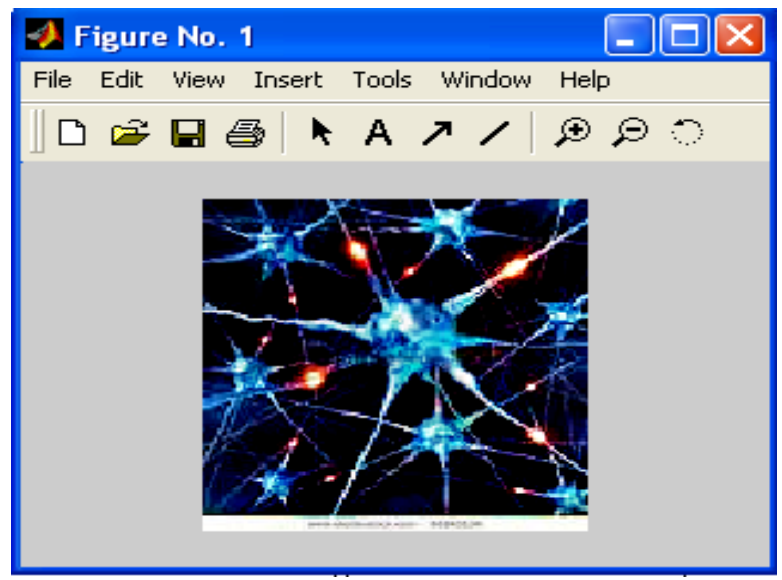

Figure 14 Subtract Images

\section{- Convert Object of Interest}

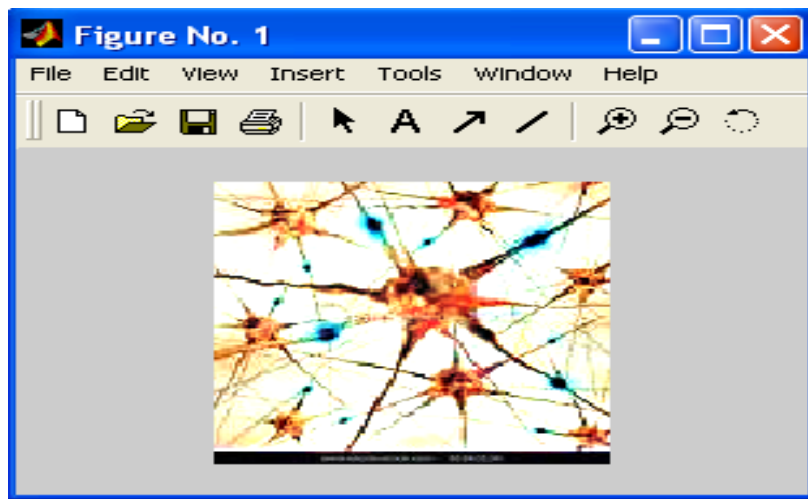

Figure 15 Convert Object of Interest

- Detect Intensity Valleys

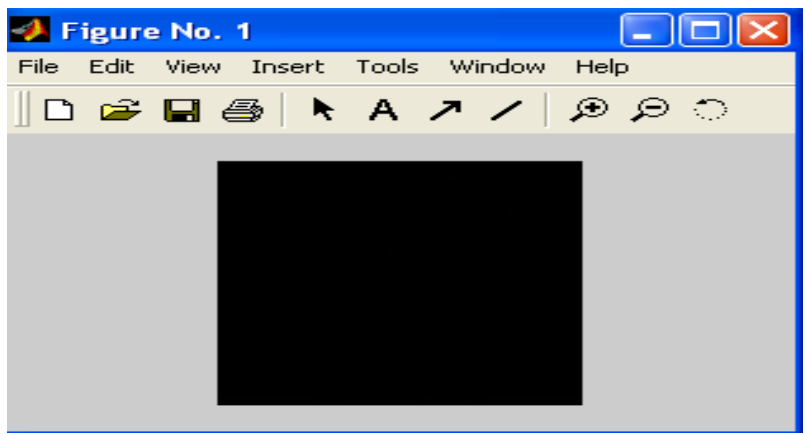

Figure 16 Detect Intensity Valleys

- Watershed Segmentation

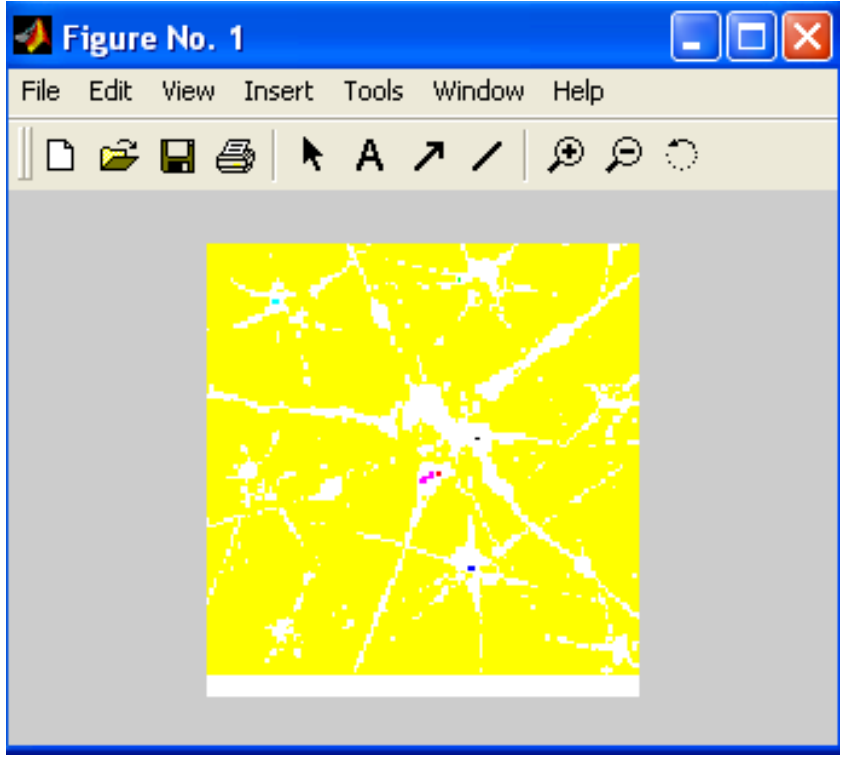

Figure 17 Watershed Segmentation 
- Extract Features from Label Matrix

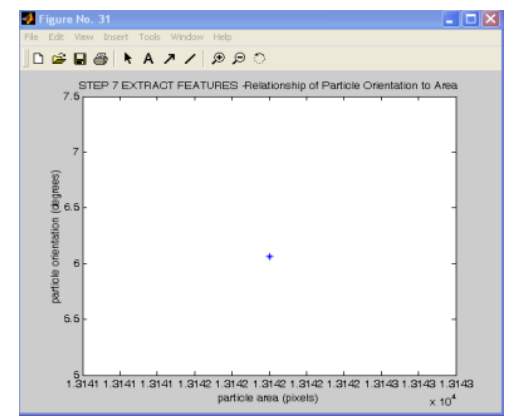

Figure 18 Extract Features from Label Matrix

\section{Conclusion}

Further studies will be devoted to track Moving neurons from Brain Video using Object Tracking algorithms and the above feature vector is classified and feed into Fuzzy logic system using Fuzzy logic tool box

\section{REFERENCES}

[1] W. Frei and C. Chen, "Fast Boundary Detection: A Generalization and New Algorithm," IEEE Trans. Computers, vol. C-26, no. 10, pp. 988-998, Oct. 1977.

[2] Kotsia, I. Pitas, I., "Facial Expression Recognition in Image Sequences Using Geometric Deformation Features and Support Vector Machines".. IEEE Trans, Image Processing, vol. 16, NO. 1, Jan. 2007

[3]http://www.cs.washington.edu/research/metip/tutor/tutor.Fi ltering.html.[4 Ziou, D. and Tabbone, S.: Edge Detection Techniques An Overview, International
Journal of Pattern Recognition and Image Analysis, 8(4):537--559, 1998

[4] Ziou, D. and Tabbone, S.: Edge Detection Techniques An Overview, International Journal of Pattern Recognition and Image Analysis, 8(4):537--559, 1998

[5] Feng-ying Cui and Li-jun Zou," Edge Feature Extraction Based on Digital Image Processing Techniques" Proceedings of the IEEE International Conference on Automation and Logistics Qingdao, China September 2008.

[6] SOBEL, I., An Isotropic $3 \times 3$ Gradient Operator, Machine Vision for Three - Dimensional Scenes, Freeman, H., Academic Pres, NY, 376-379, 1990.

[7] Ma Yan, and Zhang Zhihui, Several edge detection operators comparation, Industry and mining automation, 2004, (1): 54-56.

[8] Canny, J., A Computational Approach To Edge Detection, IEEE Trans. Pattern Analysis and Machine Intelligence, 8:679-714, 1986.

[9] S. Arivazhagan and L. Ganesan. Texture Segmentation Using Wavelet Transform. Pattern Recognition Letters, 24(16):3197-3203, December 2003.

[10] A. Gavlasov`a, A. Proch`azka, and M. Mudrov'a. Wavelet Use for Image Classification. $15^{\text {th }}$ International Conference on Process Control, 'Strbsk'e Pleso, 2005.

[11] R. C. Gonzales, R. E.Woods, and S. L. Eddins. Digital Image Processing Using MATLAB. Prentice Hall, 2004 